OPEN ACCESS

Edited by:

Jérôme Epsztein,

Institut National de la Santé et de la Recherche Médicale (INSERM),

France

Reviewed by: Christoph Schmidt-Hieber, Institut Pasteur. France Matthew Nolan, University of Edinburgh, United Kingdom

*Correspondence: Giulio Casali g.casali@ucl.ac.uk

Caswell Barry caswell.barry@ucl.ac.uk

Received: 23 August 2018 Accepted: 10 December 2018 Published: 17 January 2019

Citation:

Casali G, Shipley S, Dowell C. Hayman $R$ and Barry $C$ (2019) Entorhinal Neurons Exhibit Cue Locking in Rodent VR.

Front. Cell. Neurosci. 12:512. doi: 10.3389/fncel.2018.00512

\section{Entorhinal Neurons Exhibit Cue Locking in Rodent VR}

\author{
Giulio Casali ${ }^{1 *}$, Sarah Shipley ${ }^{1}$, Charlie Dowell ${ }^{1}$, Robin Hayman ${ }^{1,2}$ and Caswell Barry ${ }^{1 *}$ \\ ${ }^{1}$ Department of Cell and Developmental Biology, University College London, London, United Kingdom, ${ }^{2}$ Institute of \\ Neurology, University College London, London, United Kingdom
}

The regular firing pattern exhibited by medial entorhinal (mEC) grid cells of locomoting rodents is hypothesized to provide spatial metric information relevant for navigation. The development of virtual reality (VR) for head-fixed mice confers a number of experimental advantages and has become increasingly popular as a method for investigating spatiallyselective cells. Recent experiments using 1D VR linear tracks have shown that some mEC cells have multiple fields in virtual space, analogous to grid cells on real linear tracks. We recorded from the mEC as mice traversed virtual tracks featuring regularly spaced repetitive cues and identified a population of cells with multiple firing fields, resembling the regular firing of grid cells. However, further analyses indicated that many of these were not, in fact, grid cells because: (1) when recorded in the open field they did not display discrete firing fields with six-fold symmetry; and (2) in different VR environments their firing fields were found to match the spatial frequency of repetitive environmental cues. In contrast, cells identified as grid cells based on their open field firing patterns did not exhibit cue locking. In light of these results we highlight the importance of controlling the periodicity of the visual cues in VR and the necessity of identifying grid cells from real open field environments in order to correctly characterize spatially modulated neurons in VR experiments.

Keywords: grid cell, entorhinal cortex, path integration, spatial cognition, virtual navigation, virtual reality

\section{INTRODUCTION}

Since their discovery, the striking regularity of grid cell firing patterns has been proposed to play a role in encoding traveled distances and are widely held to be a core component of a circuit necessary for the integration of self-motion cues-"path integration" (Hafting et al., 2005; McNaughton et al., 2006; Burgess, 2008; Burak and Fiete, 2009; Winter et al., 2015). Equally, the influence of the sensory environment on grid cell firing is also well established. In rodents, manipulations made to familiar spatial cues result in commensurate changes to grid-patterns (Hafting et al., 2005; Barry et al., 2007; Stensola et al., 2012), in geometrically polarized environments firing is distorted (Krupic et al., 2015, 2018; Stensola et al., 2015), and in darkness their spatially periodic activity can break down completely (Chen et al., 2016; Pérez-Escobar et al., 2016).

Hence, it appears that while grid cell activity is shaped by self-motion information (Winter et al., 2015), sensory access to landmarks is necessary to maintain stable spatial firing (Hardcastle et al., 2015; Muessig et al., 2015; Campbell et al., 2018). Further, the relative efficacy of these two sources of information ("self-motion" vs. "landmark") appears to change dynamically with experience. For example, when rats first explored a pair of visually identical enclosures connected by a corridor, grid cell firing in the enclosures was highly similar, suggesting a dominance of 
landmark-based information. However, with prolonged experience, grid-patterns disambiguated the two sides, forming a single global representation of the space, consistent with increasing emphasis being placed on self-motion cues (Carpenter et al., 2015). Similarly, computational work has also highlighted the necessity of landmark information as a means to reset accumulated errors in path integration networks (Burgess and Burgess, 2014; Banino et al., 2018). However, the mechanisms by which this reset occurs and by which the relative importance of different information sources can be modulated remains unclear.

Rodent virtual reality (VR) provides a powerful and increasingly popular experimental tool capable of manipulating the characteristics of an animal's environment, thus offering an ideal means to address such issues (Thurley and Ayaz, 2017). Indeed, a number of studies have examined the responses of neurons recorded from the entorhinal and hippocampal networks in both 1D (Harvey et al., 2009; Dombeck et al., 2010; Domnisoru et al., 2013; Schmidt-Hieber and Häusser, 2013; Campbell et al., 2018) and 2D (Aronov and Tank, 2014; Chen et al., 2018). However, many cell types in these brain regions [e.g., grid cells, boundary vector cells (BVCs) and, to a certain extent, place cells] are typically identified from their open field firing patterns recorded during real environment foraging tasks. As such, it can be challenging to positively identify a neuron recorded solely from a $1 \mathrm{D}$ VR recording.

Therefore, to better understand how landmark and path integration information interact we recorded medial entorhinal (mEC) grid cells as head-fixed mice ran through three distinct 1D VR environments, each consisting of different sets of regularly repetitive spatial cues. Our rationale was to explore the effects of repetitive spatial cues on grid and non-grid cell activity. Subsequently cells were classified as grid or non-grid cells based on their activity recorded during foraging in real $2 \mathrm{D}$ environments. In the case of grid cells, we found no evidence that grid-patterns were reset to a constant phase by each cue occurrence, thus pointing to a strong influence of path integration cues. However, we identified a population of non-grid neurons that did exhibit pronounced cue-locking, firing with a constant spatial relationship to each cue occurrence. In the repetitive environments used here, these cells appeared to have strongly periodic spatial firing and were erroneously categorized as grid cells by a measure previously used to identify grid cells under such circumstances (Domnisoru et al., 2013). We highlight the importance that $2 \mathrm{D}$ recordings play in the positive identification of spatial cell types that, like grid cells, are defined by their functional properties. Moreover, the presence of a class of neurons in $\mathrm{mEC}$ responding solely to visual landmark cues is novel and raises several questions regarding their contribution to the cognitive spatial network which we aim to answer with future experiments.

\section{RESULTS}

In total, 690 unique superficial $\mathrm{mEC}$ neurons were recorded from four male C57BL/6 mice during the course of 12 experimental sessions. At the end of each recording session tetrodes were advanced by a minimum of $50 \mu \mathrm{m}$ to avoid sampling of the same cells. Post hoc examination of histology confirmed tetrode track location in superficial layers of left mEC (Supplementary Figure S1).

At the start of each recording session mice foraged in a real world (RW) 2D environment for randomly thrown drops of soya milk (SMA, Wysoy). Subsequently, after a break of at least $10 \mathrm{~min}$, they were head-fixed and then left in darkness for a further $5 \mathrm{~min}$ to habituate to the VR apparatus (see "Methods" in "Supplementary Information" and "Supplementary Figure S7"). Animals then ran in three different 1D VR environments ( $A, B$, and $C$ ) which were presented in a random order. Liquid reward was delivered at a fixed location near the end of the track (position $=875 \mathrm{~cm}$ ) after which the mice entered a black tunnel and were teleported back to the start of the track. Each environment was comprised of repeated segments containing a number of visual cues (Supplementary Figure S2). Trials in each environment were a minimum of 20 min duration. Animals showed a preference for the reward area, spending more time in the spatial bins immediately preceding the reward location than in other bins: mean \pm SEM proportion of session spent per bin (\%): reward area prior to reward $=1.46 \pm 0.03 \%$, non-reward area $=0.92 \pm 0.04 \%$, two-tailed unpaired $t$-test: $t_{(86)}=-4.24, P=5.54 \mathrm{e}^{-05}$; see Supplementary Figure S3).

In total 15 grid cells from two animals were identified based on spatial firing in the RW open field (gridness score vs. 95th percentile of a shuffled distribution, mean grid score of identified cells $=0.58 \pm 0.07$; see "Data Analysis" in "Supplementary Materials"). Visual inspection confirmed that on the VR tracks these grid cells exhibited multiple spatially localized firing fields (Figure 1A) having similar firing rates between VR and RW (mean firing rate: $\mathrm{RW}=1.3 \pm 0.2 \mathrm{~Hz}, \mathrm{VR}=1.1 \pm 0.4 \mathrm{~Hz}$, Wilcoxon signed ranked test: $P=0.07$; peak firing rate: $\mathrm{RW}=5.4 \pm 1.6 \mathrm{~Hz}, \mathrm{VR}=4.7 \pm 1.5 \mathrm{~Hz}$, Wilcoxon signed ranked test: $P=0.08$ ) but reduced stability in VR compared to RW (first vs. second half correlation: $\mathrm{RW}=0.48 \pm 0.05, \mathrm{VR}=0.07 \pm 0.03$, Wilcoxon signed ranked test: $P=0.0005)$.

Surprisingly, based on their activity in VR, relatively few of these cells were positively identified as grid cells. Specifically, using a method for grid cell categorization developed for VR environments less repetitive than the ones used here (Domnisoru et al., 2013), in environment A, 2 of 15 cells were correctly classified as grid cells, in B 5 out of 15, and in C 3 of 15 . These proportions which were not dissimilar to that of the whole ensemble of mEC cells: $\mathrm{A}=106 / 690$, binomial test: $P=0.99$, $\mathrm{B}=145 / 690$, binomial test: $P=0.17, \mathrm{C}=108 / 690$ : binomial test: $P=0.45$. Equally, the same criteria classified 74 of the 690 (10.7\%) mEC neurons as grid cells based on their 1D VR activity, only two of which were also classified as grid cells based on their RW open field activity (gridness $>95$ th of shuffle; Figure 1B; grid score $=-0.11 \pm 0.03$, stability $=0.17 \pm 0.03$, spatial information $=0.34 \pm 0.05$ ). Taken together, these numbers highlight the difficulty - and in particular high false positive rate-inherent in the identification of grid cell firing from $1 \mathrm{D}$ spatial data.

Next, we examined the extent to which grid cell activity was modulated by proximity to landmark cues in the VR. For each cell we calculated the mean spatial autocorrelogram (SAC) 
across trials in each VR environment and then detected the dominant spatial frequency-the highest peak in the $20-180 \mathrm{~cm}$ range (Figure 1C). The number of grid cells exhibiting "cue-locking" in the VR environments-having a dominant spatial frequency matching that of the cues (see "Data Analysis" in "Supplementary Materials") - was no greater than expected by chance (Figure 1D; $A=3 / 15$, binomial test: $P=0.41 ; \mathrm{B}=1 / 15$, binomial test: $P=0.14 ; \mathrm{C}=2 / 15$, binomial test: $P=0.38$ ). Indeed, 11 grid cells were not cue-locked in any of the VR environment, two were cue-locked in a single environment and two were cue-locked in two environments, with none of the grid cells being cue-locked in all three environments. Thus, grid cells-identified from the open field-showed no obvious tendency to be reset or modulated by the repetitive visual cues.

Next, we examined the spatial activity of all non-grid mEC neurons in VR $(n=675)$. To classify these neurons we developed a method analogous to a $1 \mathrm{D}$ version of the widely used gridness metric derived from the mean SAC (Sargolini et al., 2006). Briefly, cells with a prominence in the mean SAC exceeding the 95th percentile of single-cell shuffled distribution in at least two of the three VR environments were considered to exhibit significant spatially periodic activity. Our analysis
A

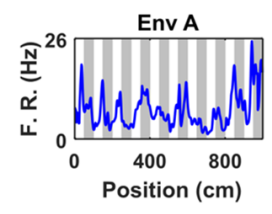

Env A

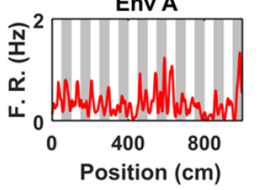

B
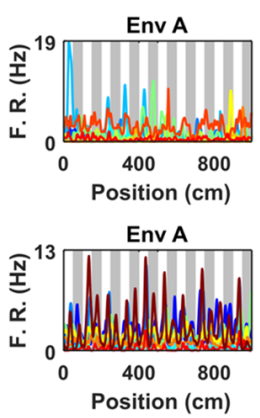

C

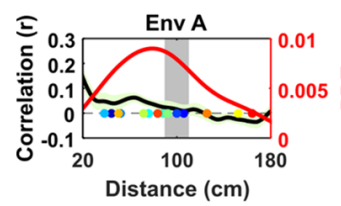

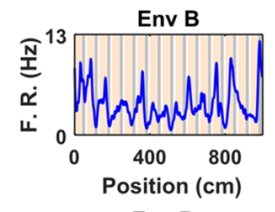

Env B

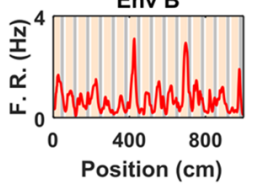

Position (cm)

\section{Grid cells from RW}

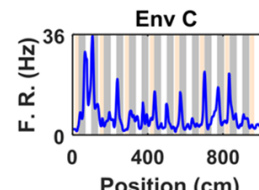

Position $(\mathrm{cm})$

Env C

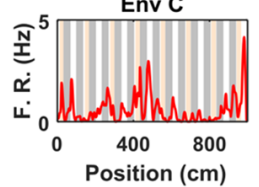

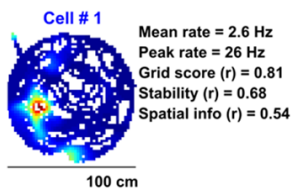

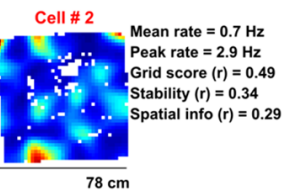

\section{Grid cells from VR}
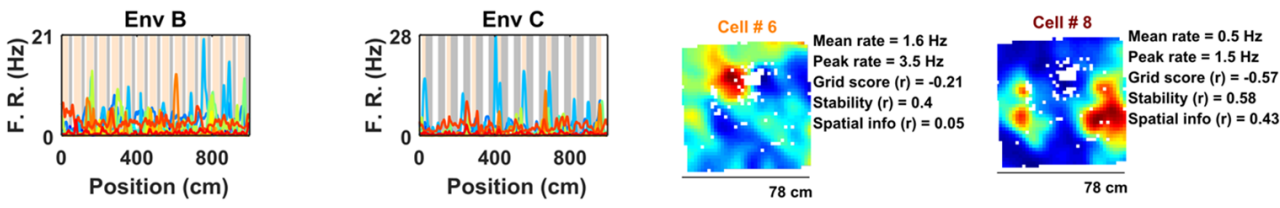

Env B
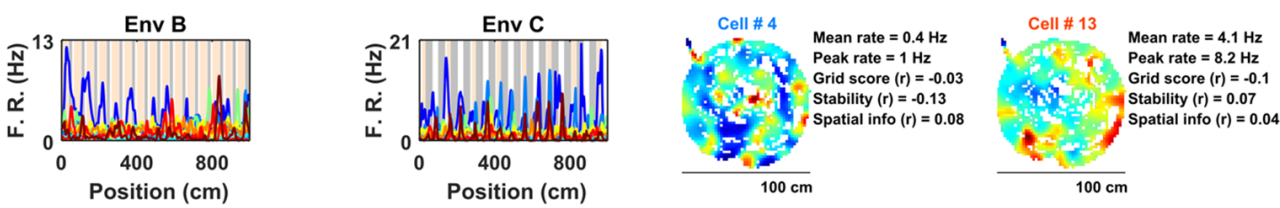

D Cue-locking
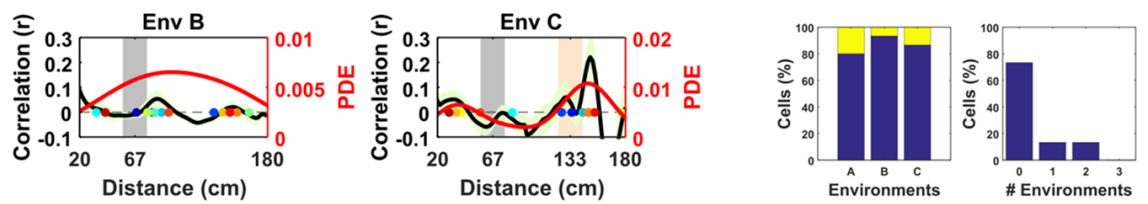

FIGURE 1 | Spatially periodic activity in real world (RW) and virtual reality (VR) environments, a subset of non-grid medial entorhinal (mEC) cells show cue-locking in VR environments. (A) Spatial activity from two example grid cells-identified based on RW recordings (right) - in each of the three VR environment (left). Repeating cues are indicated as colored bars in the background of the VR plots. Cells are color coded such that the title on RW ratemaps matches line color on the VR plots. (B) Similar to (A), spatial activity from two cells which were (incorrectly) classified as grids cells based on VR activity (left) but not based on RW open field activity (right). Despite the regularity of their firing patterns in VR, these cells showed no clear grid-like firing in RW and only limited spatial responses. (C) Cue-locking in grid cells ( $n=15$, identified from RW) was investigated using spatial auto-correlograms (SACs). Plots show mean (black line) \pm SEM (light green shade area) SACs across cells (left $y$-axis). Note the lack of periodicity corresponding to the frequency of cues in the VR environments (indicated by gray and orange bands). The overlaid color-coded dots represent the dominant spatial frequency in the 20-180 cm range detected from the SAC of each grid cell-the distribution of these points is indicated by the red line (right $y$-axis). (D) Proportion of grid cells exhibiting cue-locking (yellow) and no cue-locking (blue) in each VR environment (left) and proportion showing cue-locking in multiple VR environments (right). 


\section{Periodic cells}

\section{A $\quad \mathbf{m} 3504$}
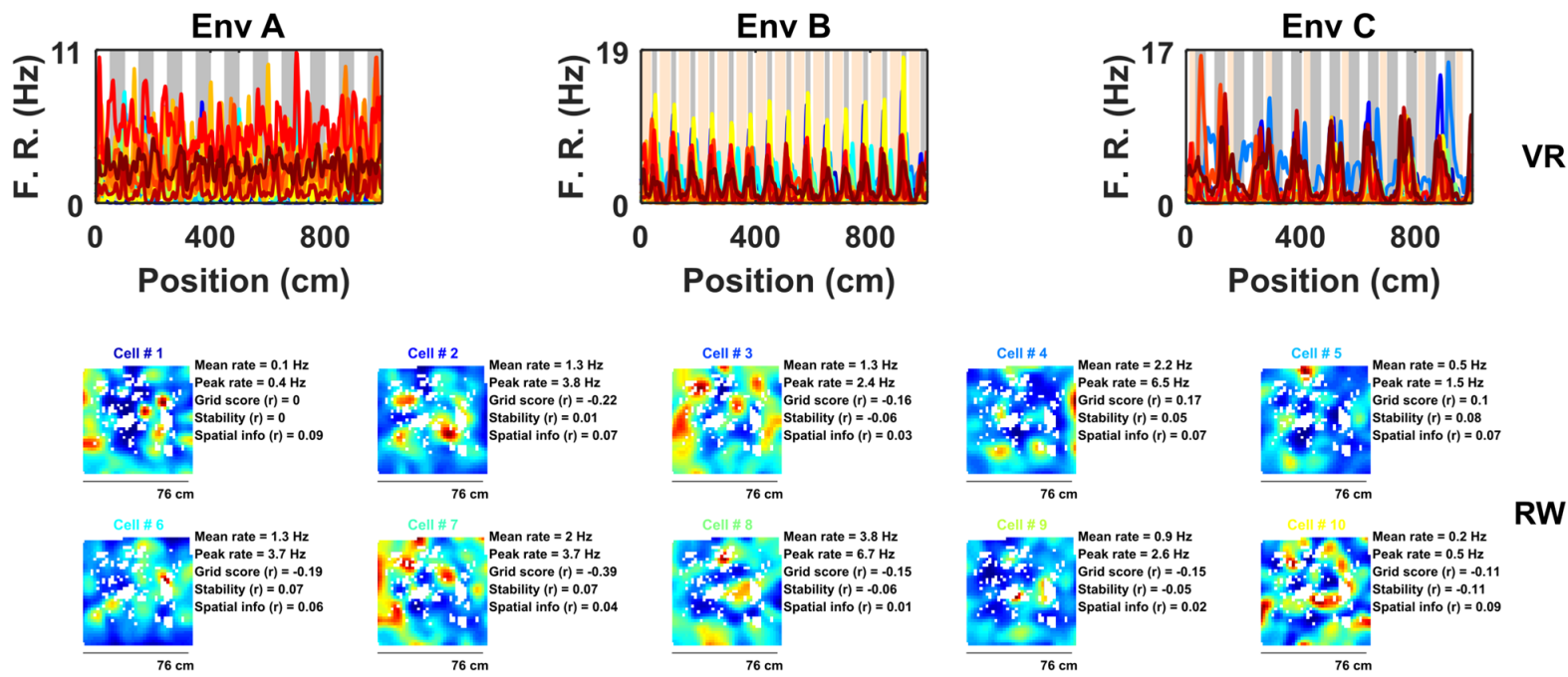

B

m3193
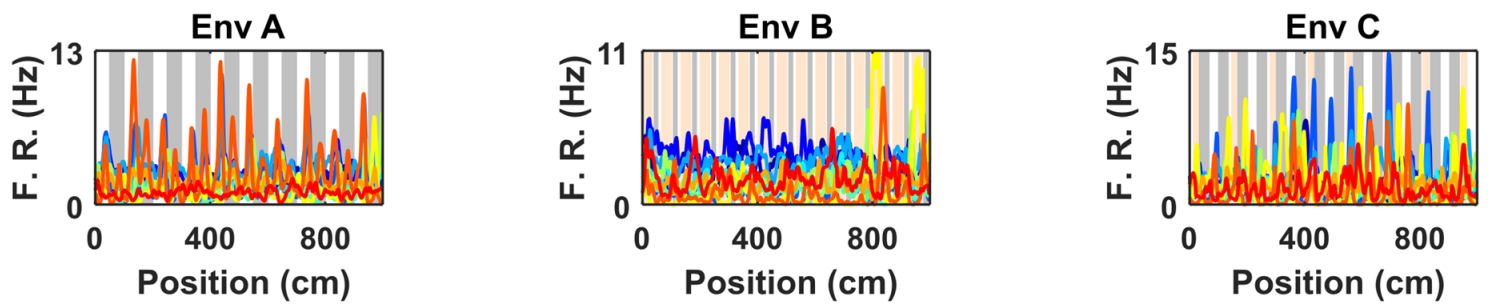

VR
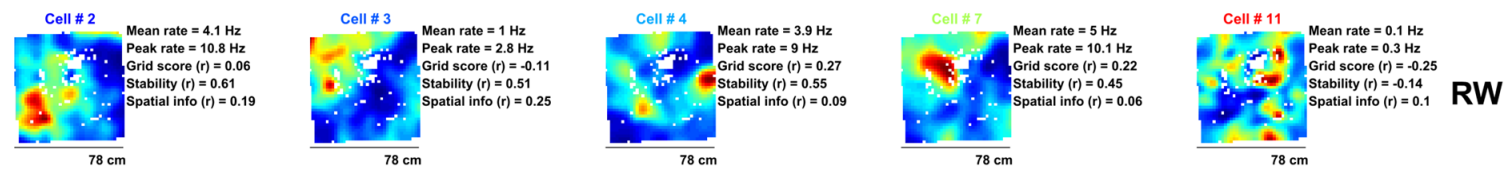

FIGURE 2 | A sub-population of non-grid cells exhibit pronounced spatial periodicity in 1D VR environments. Examples of co-recorded non-grid periodic cells from two animals (A, 10 cells from mouse m3504: B, five cells from mouse 3193) shown as ratemaps in both VR (top row) and RW (bottom rows). In VR, the firing rate of cells is plotted against position in each environment $(\mathrm{A}-\mathrm{C}$ ) with the periodicity of repeating cues indicated in the background (gray and orange). In RW the ratemaps of the same color-coded cells in VR are shown together with mean and peak firing rate, grid score, stability, and spatial information. Despite the regularity of the firing pattern patterns in VR, these cells neither showed clear grid-like firing pattern nor spatial firing of any kind in RW.

detected 56 such cells, significantly more than expected by chance (chance $=5 / 675$, binomial test, $P<0.0001$ ). Inspection of the $1 \mathrm{D}$ ratemaps of these cells revealed pronounced and regular modulation of their spatial activity across VR environments, whereas their firing patterns in RW indicated that these neurons were unlikely to be un-detected grid cells (Figure 2, Supplementary Figure S4).

Examination of their spatial firing in RW open field showed that these cells exhibited weakly spatial activity $(0.22 \pm 0.04$ bits/spike $)$ and no evidence of six-fold symmetry (0/56 cells exhibited significant grid scores vs. 95th percentile of a shuffled distribution, mean grid score $=-0.13 \pm 0.03$, spatial stability $=0.14 \pm 0.03$, Figure 2, Supplementary Figure S4). However, a large portion of these cells $(27 / 56=47 \%)$ passed criteria for grid cell inclusion based on previously used methods to detect grid cells on 1D VR tracks (Domnisoru et al., 2013).

To better characterize the nature of these non-grid periodic cells we focused on the relationship between the visual cues in $\mathrm{VR}$ and their regular firing patterns. We observed that within each VR environment the mean SAC was remarkably alike across cells, having a similar spatial frequency (Figure 3). However, the periodicity of their activity differed between environments, and in most cases coincided with the underlying spatial frequency of the repetitive cues in those environments (Figure 3A). To quantify this observation, we repeated the analysis conducted to identify modulation of grid cells by visual cues-detecting the spatial frequency of each cell from the peak in the mean SAC and comparing that with the spatial frequency 
A
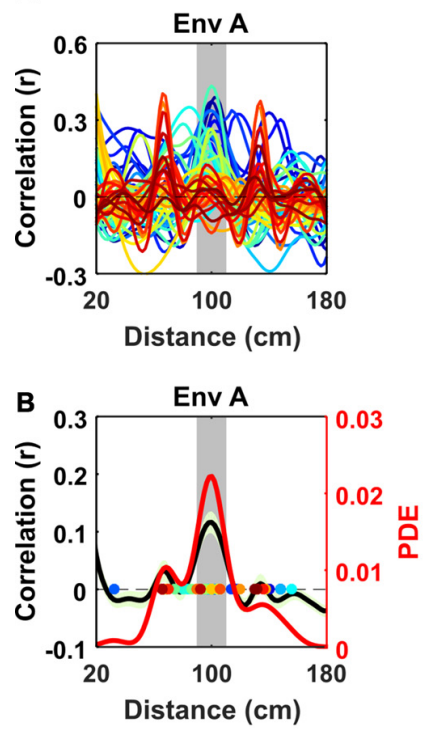

C

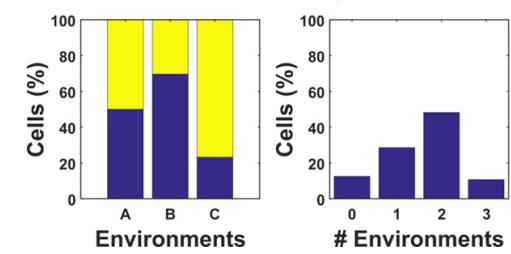

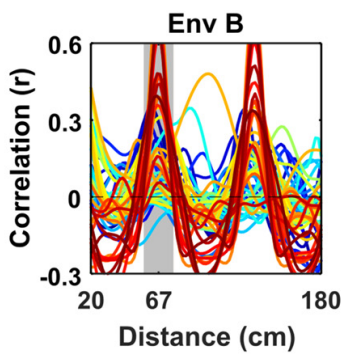
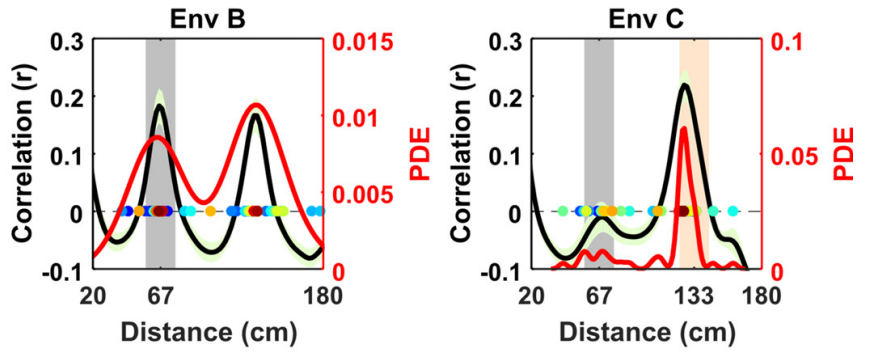

FIGURE 3 | Spatial frequency of the periodic non-grid cells. Within VR environments, periodic non-grid cells exhibited regular firing at the same spatial frequency as the underlying repetitive visual cues, unlike grid cells which showed weaker spatial periodicity of varying frequencies. (A) SAC of all non-grid periodic cells across VR environments. Note the clear peaks centered on the spatial frequency of the repeating cues of each environment (gray block for Environment $A$ and $B$ and gray and orange in Environment C). (B) Mean (black line) \pm SEM (light green shaded area) of the SAC (left $y$-axis) across cells within each environment showing clear coincidence with the frequency of the repetitive cues (gray block). Colored dots indicate the dominant spatial frequency of each cell (color matches lines in $\mathbf{A}$ ) and were used to compute the kernel density estimate (red line, right $y$-axis). (C) Histograms showing (left) percentages of non-cue locked (blue) and cue-locked (yellow) periodic cells within each VR environment. Right, Histograms showing percentages of periodic cells exhibiting cue locking in multiple environments. Most cells (>85\%) displayed cue-locking in at least one VR environments.

of cues in each environment (Figure 3B)-finding that these cells were strongly "cue-locked" (proportion cue-locked cells: environment $A=28 / 56$, binomial test: $P<0.0001 ; B=34 / 56$, $P=<0.0001 ; C=43 / 56, P<0.0001)$. Moreover, only $7 / 56$ of the periodic cells were not cue-locked in at least one of the three environments, with 16/56 being cue-locked in one environment, $27 / 56$ in two environments and 6/56 in all three environments (Figure 3C).

Together, these results suggest that the regular firing pattern exhibited by these periodic cells was strongly modulated by the repeating visual cues rather than reflecting an internallygenerated path integration signal like the one hypothesized for grid cells. In support of this notion, the proportion of periodic cells exhibiting cue-locking was significantly higher than the proportion of cue locked grid cells in environments $\mathrm{B}$ and $\mathrm{C}$ $\left(\chi^{2}=10.62, P=0.0011 ; \chi^{2}=20.5, P<0.0001\right)$ though not $\mathrm{A}$ $\left(\chi^{2}=3.71, P=0.054\right)$.

Having identified a subset of non-grid cells exhibiting strong cue modulation, we next examined how their spatial responses were distributed relative to visual cues. Since the VR environments were composed of repeating linear segments, we calculated for each cell its mean rate map over the repeating unit. Visualized in this way it was clear that the spatial periodic firing of different cue locked cells had variable phases relative to the visual cues (Figure 4A). Moreover, when rate maps were sorted according to the location of their peak activity it was apparent that there was no strong tendency for firing to cluster at specific phases of the repeated segments (Figure 4B; Rayleigh test for peak density: VR environment A: $P=0.99$, B: $P=0.99$, C: $P=0.88$ ). Finally, we examined if the relative phases at which non-periodic cells were active was conserved across environments. To test this, we focused on environments $\mathrm{A}$ and $B$ which had the simplest patterns of repetitive visual cues, and cross-correlated the stacked rate maps from both environments sorted by peak location in A (Figure 4C). The resulting crosscorrelation exhibited a single predominant peak $(r=0.25)$ which exceeded the values obtained by randomly shuffling the order of the cells before cross-correlating ( $n$ shuffles $=1,000$, peak from shuffle $r=0.014 \pm 0.002$, one-sample $t$-test, $\left.t_{(999)}=-101.1, P<0.0001\right)$. Taken together these results 

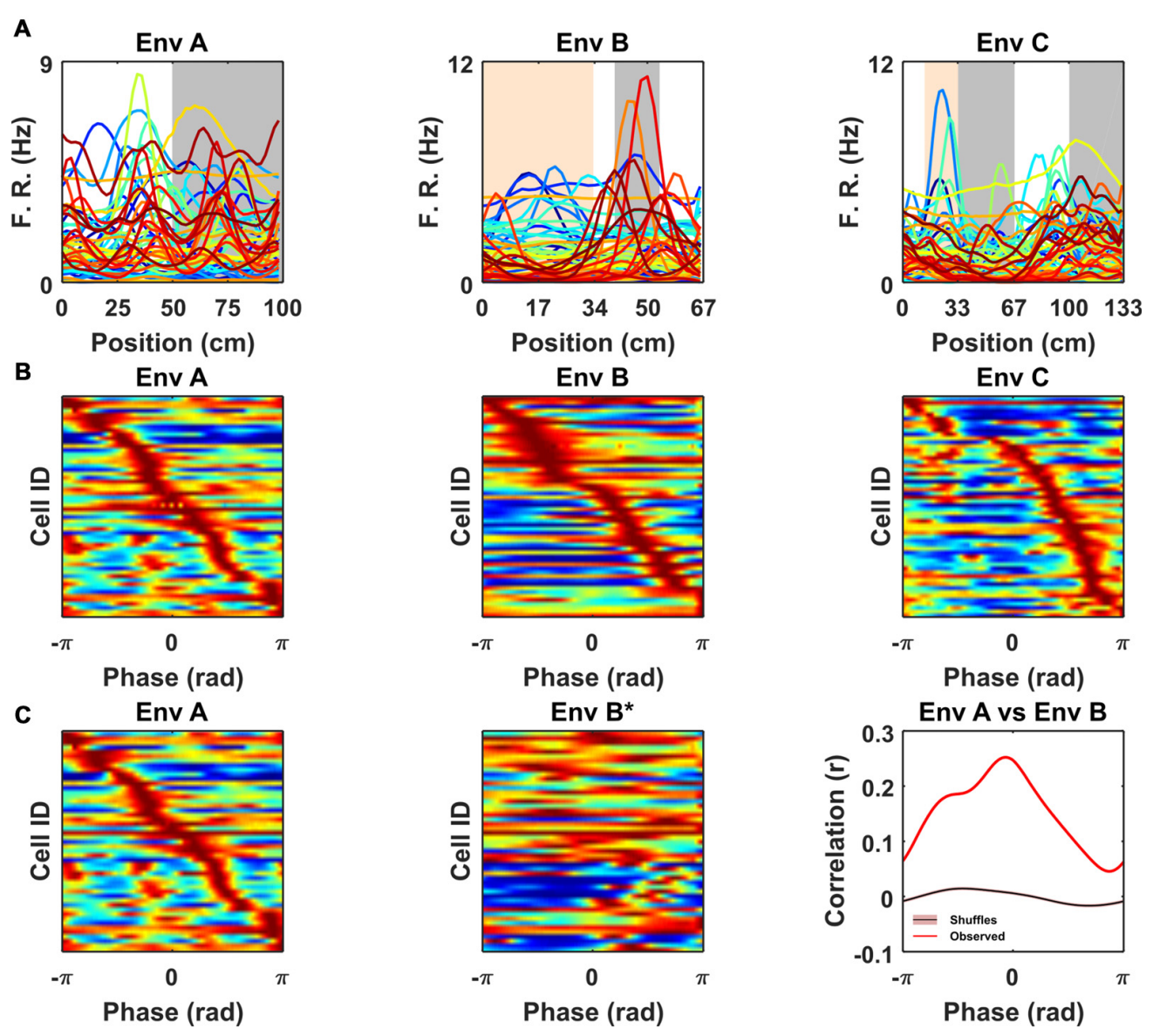

Phase (rad)

Env A vs Env B

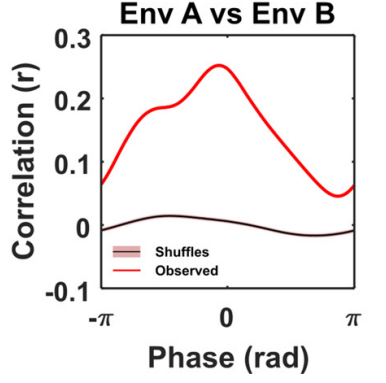

FIGURE 4 | Non-grid periodic cells were strongly modulated by the frequency of the repetitive segments in each VR but were not clustered at specific phases within each segment. (A) Rate maps for each color-coded cell across VR environments showing mean firing rate as function of location within the repeating segment. Note the differences in the peak firing rate and location of the spatial tuning curves across cells. (B) Ratemaps of all cue-locked cells sorted according to location of peak firing. For comparison across environments location within each repeated segment has been converted to a phase (radians). Note the sequence of firing within each environment with no strong preference for any particular phase. (C) Ratemaps from environments A (Left) and B* (Middle) sorted according to the order of their peaks in A. (Right) Cross-correlation between A and B* shows a significant peak (vs. 1,000 shuffles, purple line also indicates shuffle confidence interval), suggesting a tendency for the relative phase of ratemaps to be preserved between environment $A$ and $B$.

indicate that although non-grid periodic cells are strongly modulated by environmental cues there is a tendency for the relative phase at which cells fire to be preserved across environments.

\section{DISCUSSION}

The core finding presented here is the report of a distinct population of neurons in rodent $\mathrm{mEC}$ characterized by robust modulation of their firing rate by visual cues presented in linear VR environments- “cue-locked" cells. Indeed, despite the fact that the visual features differed substantially, the majority of neurons not only exhibited strong cue locking in multiple environments but also showed a marked tendency to preserve the relative sequence of their firing fields.

The observation of these cells leads to two main considerations, one with respect to how experiments are conducted in VR and one on the nature of these cells. As previously mentioned, VR has become an increasingly popular tool used to study spatial cognition and its neural basis (Harvey et al., 2009; Dombeck et al., 2010; Chen et al., 2013; Domnisoru et al., 2013; Thurley and Ayaz, 2017; Campbell et al., 2018). In particular, several studies of head-fixed mice on VR linear tracks have considered mEC neurons with multiple similarly sized firing fields to be analogous to grid cells (Domnisoru et al., 2013; Schmidt-Hieber and Häusser, 2014; Campbell et al., 2018). Clearly in linear VR environments without regularly repeating elements cue-locked cells would be expected to generate fewer repetitive fields. Nevertheless, our observation that these cells retain their firing characteristics across environments suggests they would be expected to form multiple fields under many conditions-leading them to be identified as grid cells. Importantly, these findings do not contradict conclusions drawn from previous VR grid cell studies. Indeed, many publications using $1 \mathrm{D}$ VR grid cell recordings relied on $2 \mathrm{D}$ RW environments for grid cell 
classification (Domnisoru et al., 2013; Campbell et al., 2018). Moreover, although the 1D VR environments used by Domnisoru et al. (2013) incorporated regularly repeating cues these were constrained to sub-sections of the track and were accompanied by four pairs of evenly spaced landmarks. Under such circumstances it is unlikely that cue-locking cells would be confounded with grid cells. Never-the-less, it still appears that the most reliable means of detecting grid cells is via their characteristic six-fold symmetric firing pattern visualized in a $2 \mathrm{D}$ environment either real or virtual (Hafting et al., 2005; Chen et al., 2018), though even then the expected false positive error rate is non-negligible (Barry and Burgess, 2017).

In this study, VR environments consisted of long linear tracks $(10 \mathrm{~m})$ composed of repetitive cues distributed at differing frequencies $(67,100$ and $133 \mathrm{~cm}$ ). As a consequence, the spatial activity of cue-locked cells was remarkably regular (Figure 3, Supplementary Figures S5, S6), allowing them to be identified by the strength of their periodicity. Conversely, although we observed that mEC grid cells-identified from open field trials-exhibited multiple firing fields in VR, we found no evidence for cue-locking, corroborating the widely held view that grid cells are strongly modulated by self-motion information (Hafting et al., 2005; Burgess, 2008; Carpenter et al., 2015; Winter et al., 2015; Campbell et al., 2018). In contrast, we found a population of cells showing a stable regular firing pattern during VR navigation in register with the available spatial cues. Superficially the visual pattern of the simplest environment (A) resembles drifting sine gratings. However, sensory-motor feedback between the animal's movement and the visual scene coupled with the spatial perspective and optic flow provided by other textures, suggests that the cells were responding in the context of VR navigation. In light of these considerations, we propose that the striking regular firing pattern exhibited by these cells was predominantly related to visual cues, and hence we consider them to be "cue-locked." We note that these cells' spatial fields were not limited to the immediate proximity of cues but spread across the range of possible phases within each repeated environmental segment (Figure 4, Supplementary Figures S5, S6) in a way that resembled the sequential firing of an ensemble of grid cells from the same module (Barry et al., 2007; Stensola et al., 2012; Yoon et al., 2016). Interestingly, our results suggest that such a sequential firing pattern was conserved at least between two VR environments (Figures 4A,B), suggesting that cue-locking cells may encode relative distances from the cues. In VR, CA1 pyramidal cells have been seen to exhibit spatial activity that is strongly modulated by distinct visual cues (Bourboulou et al., 2018) and similarly, even in the open field, place cell firing can be controlled by individual features of an environment (Fenton et al., 2000; Rivard et al., 2004). It seems plausible then that the responses we report in superficial $\mathrm{mEC}$ may contribute in part or indeed wholly to those noted in the downstream hippocampus.

What might be the identity of the cue-locked cells? One possible interpretation is that these neurons are BVCs (Hartley et al., 2000; Barry et al., 2006; Lever et al., 2009) or border cells (Solstad et al., 2008) responding to visual cues that are perceived as a boundary. However, the lack of clear spatial modulation in the open field trials renders this unlikely as BVC firing fields in the open field are typically expected to be unitary and elongated-a simple function of the animals allocentric location relative to environmental boundaries (Hartley et al., 2000; Lever et al., 2009). Equally it is possible that a subset of these cue-locked cells may be non-grid spatial cells (Diehl et al., 2017), though again, the lack of clear spatial modulation in the open field would suggest otherwise. Without further evidence it is hard to draw solid conclusions. Still it seems plausible that these cells respond to visual features of intermediate complexity and may likely be modulated by the egocentric location of the cue relative to the animal. Although open field recording enclosures often include only a small number of controlled cues like a cue card, it is likely they also include a large number of uncontrolled cues which are unintentionally present. Due to their nature it is necessarily hard to quantify the prevalence and efficacy of such uncontrolled cues. These considerations would account for their relatively simple firing characteristics in the VR-which is both sensorially impoverished and behaviorally constrained-and more complex activity during open field foraging.

Therefore, it falls to future work to further characterize the activity of these cells and the factors they respond to. On one hand it is obvious that the highly simplified and well controlled environments provided by VR have a role in this endeavor but equally less constrained open field recordings combined with careful behavioral tracking will also be important.

\section{ETHICS STATEMENT}

All procedures were licensed by the UK Home Office following the revised ASPA regulations (2013) modified by the European Directive 2010/63/EU.

\section{AUTHOR CONTRIBUTIONS}

CB conceived the study and obtained funding. GC, SS and $\mathrm{CB}$ designed the protocol. GC, SS and $\mathrm{RH}$ performed surgeries. GC, SS and CD conducted experiments. GC analyzed data. All authors interpreted data and discussed results. GC and CB wrote the manuscript. All authors commented and edited the manuscript.

\section{FUNDING}

This work was supported by grants from the Wellcome Trust Sir Henry Dale and Royal Society.

\section{SUPPLEMENTARY MATERIAL}

The Supplementary Material for this article can be found online at: https://www.frontiersin.org/articles/10.3389/fncel. 2018.00512/full\#supplementary-material 


\section{REFERENCES}

Aronov, D., and Tank, D. W. (2014). Engagement of neural circuits underlying 2D spatial navigation in a rodent virtual reality system. Neuron $84,442-456$. doi: 10.1016/j.neuron.2014.08.042

Banino, A., Barry, C., Uria, B., Blundell, C., Lillicrap, T., Mirowski, P., et al. (2018). Vector-based navigation using grid-like representations in artificial agents. Nature 557, 429-433. doi: 10.1038/s41586-018-0102-6

Barry, C., and Burgess, N. (2017). To be a grid cell: shuffling procedures for determining "gridness". BioRxiv 230250 doi: 10.1101/230250

Barry, C., Hayman, R., Burgess, N., and Jeffery, K. J. (2007). Experience-dependent rescaling of entorhinal grids. Nat. Neurosci. 10, 682-684. doi: 10.1038/nn1905

Barry, C., Lever, C., Hayman, R., Hartley, T., Burton, S., O’Keefe, J., et al. (2006). The boundary vector cell model of place cell firing and spatial memory. Rev. Neurosci. 17, 71-97. doi: 10.1515/revneuro.2006.17.1-2.71

Bourboulou, R., Marti, G., Michon, F.-X., Nouguier, M., Robbe, D., Koenig, J., et al. (2018). Dynamic control of hippocampal spatial coding resolution by local visual cues. BioRxiv 275420 [Preprint]. doi: 10.1101/275420

Burak, Y., and Fiete, I. R. (2009). Accurate path integration in continuous attractor network models of grid cells. PLoS Comput. Biol. 5:e1000291. doi: 10.1371/journal.pcbi.1000291

Burgess, N. (2008). Grid cells and theta as oscillatory interference: theory and predictions. Hippocampus 18, 1157-1174. doi: 10.1002/hipo.20518

Burgess, C. P., and Burgess, N. (2014). Controlling phase noise in oscillatory interference models of grid cell firing. J. Neurosci. 34, 6224-6232. doi: 10.1523/jneurosci.2540-12.2014

Campbell, M. G., Ocko, S. A., Mallory, C. S., Low, I. I. C., Ganguli, S., and Giocomo, L. M. (2018). Principles governing the integration of landmark and self-motion cues in entorhinal cortical codes for navigation. Nat. Neurosci. 21, 1096-1106. doi: 10.1038/s41593-018-0189-y

Carpenter, F., Manson, D., Jeffery, K., Burgess, N., and Barry, C. (2015). Grid cells form a global representation of connected environments. Curr. Biol. 25, 1176-1182. doi: 10.1016/j.cub.2015.02.037

Chen, G., King, J. A., Burgess, N., and O'Keefe, J. (2013). How vision and movement combine in the hippocampal place code. Proc. Natl. Acad. Sci. U S A 110, 378-383. doi: 10.1073/pnas.1215834110

Chen, G., King, J. A., Lu, Y., Cacucci, F., and Burgess, N. (2018). Spatial cell firing during virtual navigation of open arenas by head-restrained mice. Elife 7:e34789. doi: 10.7554/eLife.34789

Chen, G., Manson, D., Cacucci, F., and Wills, T. J. (2016). Absence of visual input results in the disruption of grid cell firing in the mouse. Curr. Biol. 26, 2335-2342. doi: 10.1016/j.cub.2016.06.043

Diehl, G. W., Hon, O. J., Leutgeb, S., and Leutgeb, J. K. (2017). Grid and nongrid cells in medial entorhinal cortex represent spatial location and environmental features with complementary coding schemes. Neuron 94, 83.e6-92.e6. doi: 10.1016/j.neuron.2017.03.004

Dombeck, D. A., Harvey, C. D., Tian, L., Looger, L. L., and Tank, D. W. (2010). Functional imaging of hippocampal place cells at cellular resolution during virtual navigation. Nat. Neurosci. 13, 1433-1440. doi: 10.1038/nn.2648

Domnisoru, C., Kinkhabwala, A. A., and Tank, D. W. (2013). Membrane potential dynamics of grid cells. Nature 495, 199-204. doi: 10.1038/nature11973

Fenton, A. A., Csizmadia, G., and Muller, R. U. (2000). Conjoint control of hippocampal place cell firing by two visual stimuli. J. Gen. Physiol. 116, 191-210. doi: 10.1085/jgp.116.2.191

Hafting, T., Fyhn, M., Molden, S., Moser, M.-B., and Moser, E. I. (2005). Microstructure of a spatial map in the entorhinal cortex. Nature 436, 801-806. doi: $10.1038 /$ nature 03721

Hardcastle, K., Ganguli, S., and Giocomo, L. M. (2015). Environmental boundaries as an error correction mechanism for grid cells. Neuron 86, 827-839. doi: 10.1016/j.neuron.2015.03.039

Hartley, T., Burgess, N., Lever, C., Cacucci, F., and O'Keefe, J. (2000). Modeling place fields in terms of the cortical inputs to the hippocampus. Hippocampus 10, 369-379. doi: 10.1002/1098-1063(2000)10:4<369::AID-HIPO3>3.0.CO;2-0
Harvey, C. D., Collman, F., Dombeck, D. A., and Tank, D. W. (2009). Intracellular dynamics of hippocampal place cells during virtual navigation. Nature 461, 941-946. doi: 10.1038/nature08499

Krupic, J., Bauza, M., Burton, S., Barry, C., and O'Keefe, J. (2015). Grid cell symmetry is shaped by environmental geometry. Nature 518, 232-235. doi: 10.1038/nature14153

Krupic, J., Bauza, M., Burton, S., and O'Keefe, J. (2018). Local transformations of the hippocampal cognitive map. Science 359, 1143-1146. doi: 10.1126/science. aao4960

Lever, C., Burton, S., Jeewajee, A., O’Keefe, J., and Burgess, N. (2009). Boundary vector cells in the subiculum of the hippocampal formation. J. Neurosci. 29, 9771-9777. doi: 10.1523/JNEUROSCI.1319-09.2009

McNaughton, B. L., Battaglia, F. P., Jensen, O., Moser, E. I., and Moser, M.-B. (2006). Path integration and the neural basis of the "cognitive map". Nat. Rev. Neurosci. 7, 663-678. doi: 10.1038/nrn1932

Muessig, L., Hauser, J., Wills, T. J., and Cacucci, F. (2015). A developmental switch in place cell accuracy coincides with grid cell maturation. Neuron 86, 1167-1173. doi: 10.1016/j.neuron.2015.05.011

Pérez-Escobar, J. A., Kornienko, O., Latuske, P., Kohler, L., and Allen, K. (2016). Visual landmarks sharpen grid cell metric and confer context specificity to neurons of the medial entorhinal cortex. Elife 5:e16937. doi: 10.7554/elife. 16937

Rivard, B., Li, Y., Lenck-Santini, P.-P., Poucet, B., and Muller, R. U. (2004). Representation of objects in space by two classes of hippocampal pyramidal cells. J. Gen. Physiol. 124, 9-25. doi: 10.1085/jgp.200409015

Sargolini, F., Fyhn, M., Hafting, T., McNaughton, B. L., Witter, M. P., Moser, M.-B., et al. (2006). Conjunctive representation of position, direction and velocity in entorhinal cortex. Science 312, 758-762. doi: 10.1126/science. 1125572

Schmidt-Hieber, C., and Häusser, M. (2013). Cellular mechanisms of spatial navigation in the medial entorhinal cortex. Nat. Neurosci. 16, 325-331. doi: $10.1038 / \mathrm{nn} .3340$

Schmidt-Hieber, C., and Häusser, M. (2014). How to build a grid cell. Philos. Trans. R. Soc. Lond. B Biol. Sci. 369:20120520. doi: 10.1098/rstb.2012.0520

Solstad, T., Boccara, C. N., Kropff, E., Moser, M.-B., and Moser, E. I. (2008). Representation of geometric borders in the entorhinal cortex. Science 322, 1865-1868. doi: 10.1126/science.1166466

Stensola, T., Stensola, H., Moser, M.-B., and Moser, E. I. (2015). Shearinginduced asymmetry in entorhinal grid cells. Nature 518, 207-212. doi: $10.1038 /$ nature 14151

Stensola, H., Stensola, T., Solstad, T., Frøland, K., Moser, M.-B., and Moser, E. I. (2012). The entorhinal grid map is discretized. Nature 492, 72-78. doi: $10.1038 /$ nature 11649

Thurley, K., and Ayaz, A. (2017). Virtual reality systems for rodents. Curr. Zool. 63, 109-119. doi: 10.1093/cz/zow070

Winter, S. S., Mehlman, M. L., Clark, B. J., and Taube, J. S. (2015). Passive transport disrupts grid signals in the parahippocampal cortex. Curr. Biol. 25, 2493-2502. doi: 10.1016/j.cub.2015.08.034

Yoon, K., Lewallen, S., Kinkhabwala, A. A., Tank, D. W., and Fiete, I. R. (2016). Grid cell responses in 1D environments assessed as slices through a $2 \mathrm{D}$ lattice. Neuron 89, 1086-1099. doi: 10.1016/j.neuron.2016.01.039

Conflict of Interest Statement: The authors declare that the research was conducted in the absence of any commercial or financial relationships that could be construed as a potential conflict of interest.

Copyright (c) 2019 Casali, Shipley, Dowell, Hayman and Barry. This is an open-access article distributed under the terms of the Creative Commons Attribution License (CC BY). The use, distribution or reproduction in other forums is permitted, provided the original author(s) and the copyright owner(s) are credited and that the original publication in this journal is cited, in accordance with accepted academic practice. No use, distribution or reproduction is permitted which does not comply with these terms. 